\title{
Children with Acute Neurological Emergency
}

\author{
Gunes Orman, Andrea Rossi, Avner Meoded, \\ and Thierry A. G. M. Huisman
}

\section{Learning Objectives}

- To understand common acute pediatric neurological disorders that are encountered in the emergency setting.

- To understand basic neuroimaging findings of acute pediatric neurological emergencies.

Children with acute neurological emergencies frequently present to the emergency room (ER) with various combinations of headache, fever, nausea, vomiting, physical signs of trauma, altered mental status, coma, or focal neurological symptoms. Neuroimaging is often needed in children with acute neurological emergencies due to the difficulties of gathering an adequate and comprehensive patient history or a detailed and reliable neurological examination [1]. The radiologist plays an important role in the ER. Firstly, the radiologist should be able to answer if the child needs neuroimaging emergently. Secondly, the radiologist should be able to answer which imaging modality is appropriate for the child. Different imaging modalities may be chosen based on the institutional availability and clinical status of the patient. Computed tomography (CT) and magnetic resonance imaging (MRI) are the most frequently used imaging modalities in the emergency setting. CT is usually the initial modality of choice in most of the situations. Modern multidetector CT scanners render highly detailed multiplanar submillimeter images of the pediatric brain; however, the main disadvantage of $\mathrm{CT}$ is that it requires

G. Orman $(\bowtie) \cdot$ A. Meoded · T. A. G. M. Huisman Edward B. Singleton Department of Radiology, Texas Children's Hospital, Houston, TX, USA

e-mail: gxorman@texaschildrens.org;

axmeoded@texaschildrens.org; huisman@texaschildrens.org

A. Rossi

Neuroradiology Unit, IRCCS Istituto Giannina Gaslini,

Genoa, Italy

e-mail: andrearossi@gaslini.org the use of ionizing radiation [2]. MRI is a valuable tool to confirm and characterize the entities seen in children with acute neurological emergency. MRI is easy to perform, does not use ionizing radiation, offers multiple different tissue contrasts, superior anatomical details, especially for evaluation of the posterior fossa, and offers multiple advanced, and functional sequences enhancing a sensitive and specific diagnosis $[2,3]$. These advantages made MRI an increasingly preferred modality for the diagnostic evaluation of children with acute neurological symptoms even in time-sensitive settings. Ultrafast MRI protocols should be considered whenever possible as the initial choice of imaging instead of CT [3, 4]. Advanced MRI techniques including susceptibility-weighted imaging (SWI), diffusion weighted imaging (DWI), or diffusion tensor imaging (DTI) with apparent diffusion coefficient (ADC) mapping and perfusion-weighted imaging (PWI) are progressively used in acute pediatric neuroimaging protocols [2]. Last but not least, the radiologist should be able to give a reliable differential diagnosis to guide patient management, in order to reduce morbidity and mortality. In addition, when evaluating brain MRIs of children, the radiologist should be aware that the pediatric brain may be immature and depending on the child's age different states of brain development and maturation may be encountered. A neonatal brain MRI looks very different compared to a teenager's brain MRI. Thus, the radiologist should understand normal brain development and must be familiar with age-specific changes of the developing pediatric brain [2].

The goal of this chapter is to discuss the neuroimaging findings of a number of common acute pediatric neurological emergencies including stroke, hemorrhage, infections, and several less frequently encountered disorders.

\subsection{Stroke}

Arterial ischemic stroke (AIS) is relatively rare in children but can lead to significant morbidity and mortality rates ranging between $4 \%$ and $14 \%$. Understanding that children with AIS 
present clinically differently than adults and often present with unique child specific risk factors will impact the diagnostic approach, treatment, and outcome. The risk factors for AIS in children include cardiac disorders, infection, head and neck trauma, sickle cell disease, vascular malformations, genetic disorders, and autoimmune diseases [5-7]. The clinical presentation in neonates and infants can be very subtle relative to the severity of imaging findings (Fig. 14.1a). In children with suspected stroke, MRI is the preferred imaging modality. Radiologists should embrace an "MRI first" service whenever possible. Recommended sequences include DWI/DTI and ADC mapping to diagnose acute ischemia, gradient-recalled echo (GRE) or SWI to detect intracranial blood and its breakdown products, and T1-weighted imaging (WI) and T2-WI to assess for myelination, extra-axial blood, and edema [8].

Furthermore, combined analysis of DWI/PWI data can identify tissue at risk of progression to infarction, e.g., "ischemic penumbra" [9]. The penumbra shows critical hypoperfusion without matching diffusion restriction (DWI/PWI mismatch), as opposed to the ischemic core represented by matching areas of hypoperfusion and restricted diffusion. Early and reliable identification of potentially salvageable brain tissue is essential and could serve as a guide to treatment [10].

In addition, simultaneous imaging of the head and neck vasculature should always be considered/included in the acute workup if possible. Magnetic resonance angiography (MRA) of the brain is useful to detect vascular abnormalities (Fig. 14.1b). In children with AIS rapid treatment with neuroprotection, antithrombotic medications, and potential use of tissue plasminogen activator (tPA) are recommended to limit infarct progression/severity and stroke recurrence [11].

Hemorrhagic venous stroke (VS) may occur as complication of cerebral venous sinus thrombosis (CVST). The most common predisposing factors are systemic or local infection, hypercoagulable state, and dehydration [1]. CVST should be suspected in any child with hemorrhage or brain injury that cannot be explained by an arterial distribution of infarction or history of trauma or infection. If there are features of hemorrhage, additional MR venography (MRV) may be needed to provide diagnostic confidence (Fig. 14.1c). Post-contrast 3D-T1-WI ultrafast GRE sequence may also be helpful to detect venous filling defects [12]. CVST most frequently affects the superior sagittal sinus followed by the transverse sinus, with resulting VS mostly in these venous drainage regions. VS may not demonstrate the same degree of restricted diffusion as AIS due to associated vasogenic edema. Physicians should hold an increased level of clinical suspicion especially in children with risk factors [1]. Rapid imaging and correct diagnosis is very important.

Clinical mimickers of pediatric stroke include systemic hypoglycemia, hemiplegic migraine, seizure, postictal paresis, acute disseminated encephalomyelitis (ADEM), infection and may be seen in up to $21 \%$ of children with the initial clinical suspicion of an acute stroke [12-14]. The frequency of these disorders underlines the value of MRI in the emergent evaluation of suspected stroke [12].

\section{Key Point}

- Neuroimaging is extremely time sensitive in children suspected of arterial or venous stroke. MRI with DWI/DTI and ADC is the established imaging modality of choice for the diagnosis.

\subsection{Hemorrhages}

Intracranial hemorrhages ( $\mathrm{ICH})$ are a frequent cause of severe neurological morbidity and mortality in children. Excluding head trauma which is an important cause of $\mathrm{ICH}$, other causes of spontaneous ICH include arteriovenous malformations (AVM), aneurysms, cavernous malformations, hematological disorders, and brain tumors in children. In addition, ICH may be observed as a complication of AIS and VS. Children may present with headache, vomiting, impaired consciousness, convulsions, and focal neurological deficits. Spontaneous ICH can also present with non-specific symptoms in children especially younger than 3 years of age. Subacute courses are common and may result in a diagnostic delay which may result in a higher mortality and morbidity, especially for infratentorial hemorrhages, in aneurysms, children younger than 3 years and children with underlying hematological disorders [15].

ICH imaging appearances follow a well-known evolution with five distinct phases identified: hyperacute, acute, early subacute, late subacute, and chronic. Neuroimaging findings and appearance vary with the phase (Table 14.1) [16]. On CT, early hyperacute ICH are isodense to normal brain, could be missed if not carefully observed. However, multiplanar reconstructed CT images increase the sensitivity of CT for detection of ICH [17]. Acute and early subacute ICH reveal hyperdensity due to progressive blood clot retraction, while progressive red blood cell lysis decreases the density in the late subacute phase. In the chronic phase, progressive blood resorption results in a hypodense, cerebrospinal fluid (CSF)-filled brain defect. Similar temporal signal changes are observed for the various MRI sequences (Fig. 14.2). The imaging characteristics of $\mathrm{ICH}$ are summarized in Table 14.1. T2*, GRE, or SWI sequences are most sensitive to blood products and should be included in the MRI protocol when ICH are suspected in the emergency setting. Neuroimaging should not only focus on diagnosis of $\mathrm{ICH}$ and its complications but should also focus on finding the underlying cause of the ICH. MRA and MRV sequences and contrast-enhanced MRI may be necessary to exclude the underlying cause such as vascular malformations and tumors [16]. 

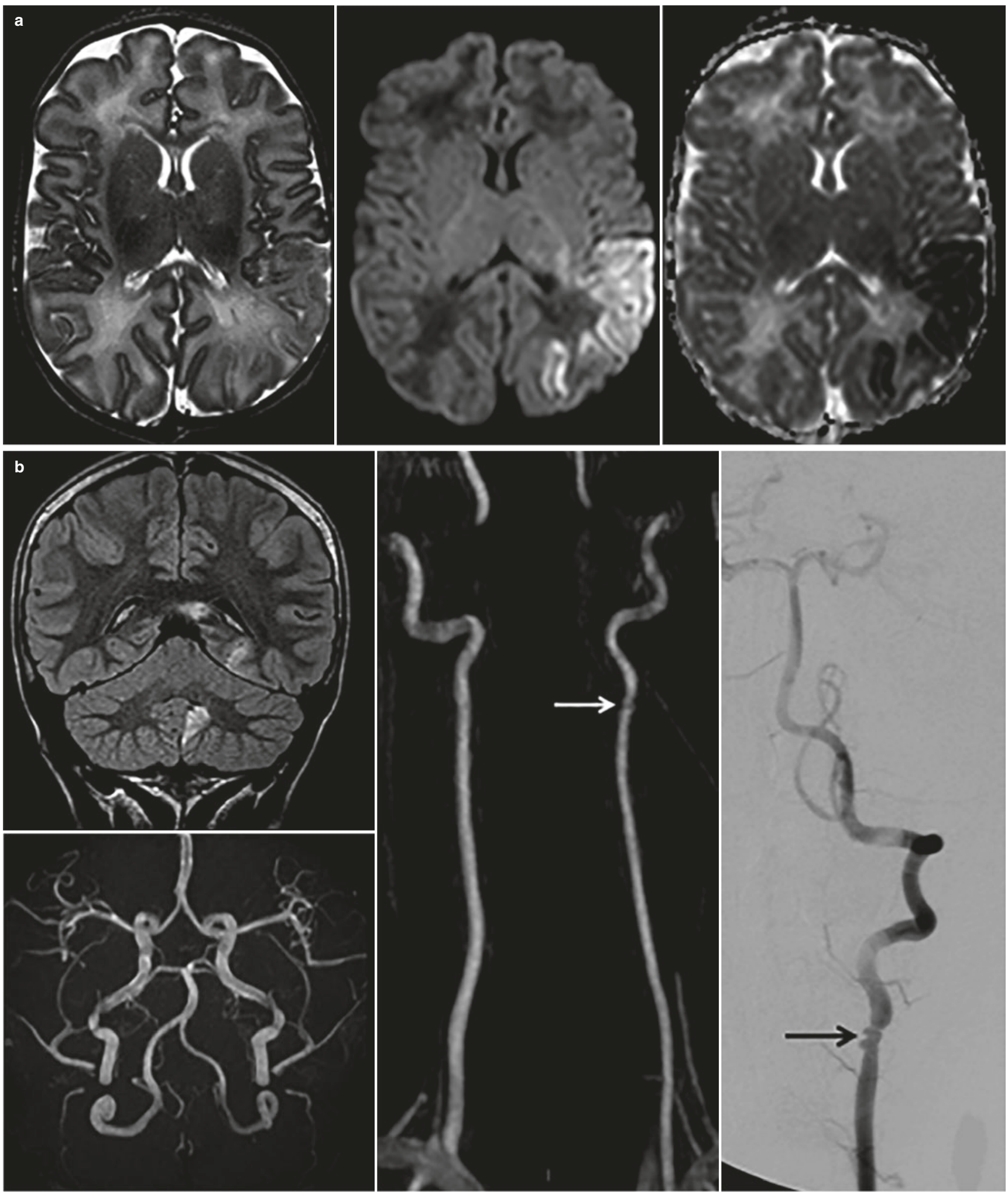

Fig. 14.1 (a, top row) In this 25-day old girl with prior surgery for intestinal atresia and recent onset of clonic fits involving the right limbs, axial T2-weighted MRI shows ill-defined cortico-subcortical blurring of the left parietal lobe. Trace DWI and corresponding ADC map clearly reveal restricted diffusion consistent with an acute ischemic stroke involving the posterior portion of the left MCA territory. (b, middle two rows) In this 13-year old boy with a subacute presentation with ataxia, coronal FLAIR shows multiple hyperintense areas involving the cerebellum and occipital lobe, suggesting a thromboembolic mechanism involving the posterior circulation. Intracranial TOF MR angiography is normal. Cervical
CE-MR angiography, however, shows focal irregularity (arrow) of the left vertebral artery which was confirmed by catheter angiography showing a string-of-beads pattern (arrow) consistent with the eventual diagnosis of fibromuscular dysplasia. (c, lower row) In this 16-year-old girl with recent posterior fossa surgery, sagittal T2-W MRI shows deceivingly normal appearance of the superior sagittal sinus, due to hypointense acute thrombus. Contrast-enhanced axial T1-weighted MRI shows the empty delta sign (arrow) consistent with intraluminal sinus thrombosis, which is confirmed by phase contrast MR venography showing absent flow signal involving the totality of the superior sagittal and straight sinuses 

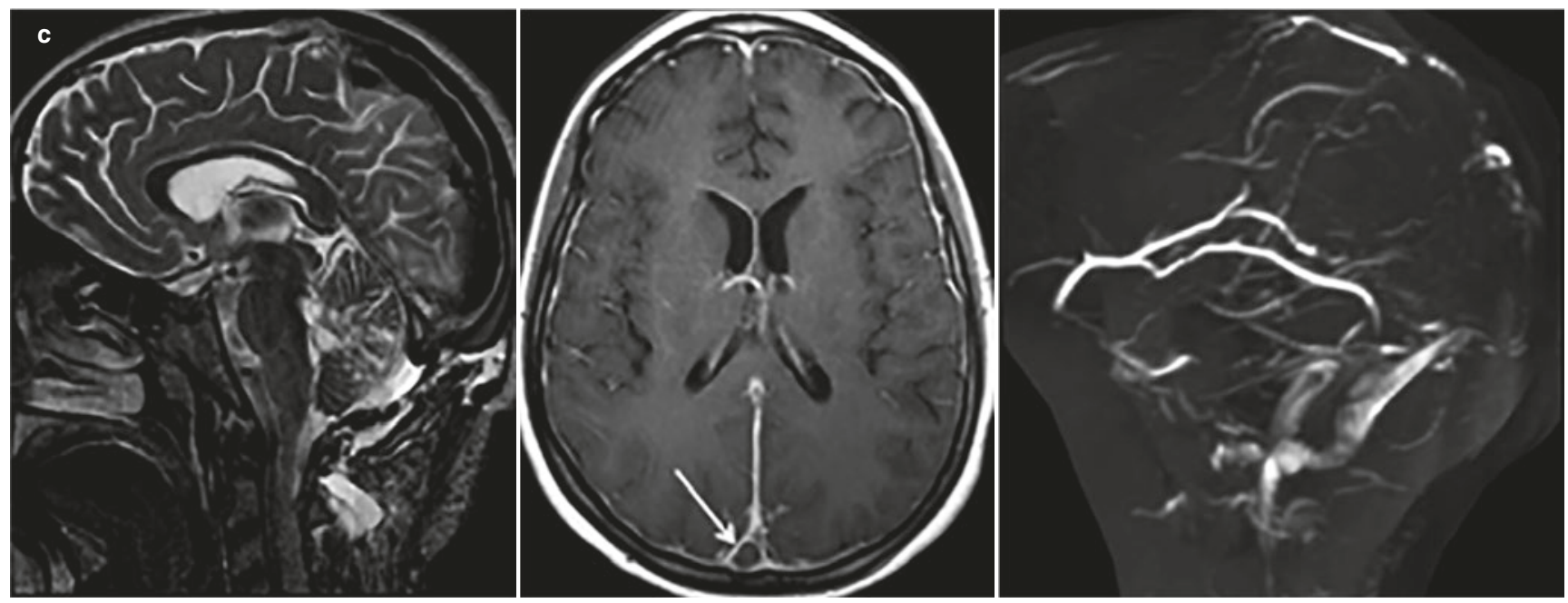

Fig. 14.1 (continued)

Table 14.1 Evolution of ICH characteristics on CT and MRI

\begin{tabular}{|c|c|c|c|}
\hline $\begin{array}{l}\text { Hematoma } \\
\text { age/phase }\end{array}$ & CT & $\begin{array}{l}\text { T1-weighted } \\
\text { MRI }\end{array}$ & T2-weighted MRI \\
\hline $\begin{array}{l}\text { Hyperacute } \\
(<12 \mathrm{~h})\end{array}$ & Isodense & $\begin{array}{l}\text { T1-iso/ } \\
\text { hypointense }\end{array}$ & T2-hyperintense \\
\hline $\begin{array}{l}\text { Acute } \\
(12 \mathrm{~h}-2 \mathrm{~d})\end{array}$ & $\begin{array}{l}\text { Increasing } \\
\text { density }\end{array}$ & $\begin{array}{l}\text { T1-iso/ } \\
\text { hypointense }\end{array}$ & T2-hypointense \\
\hline $\begin{array}{l}\text { Early } \\
\text { subacute } \\
(2-7 d)\end{array}$ & $\begin{array}{l}\text { Increased } \\
\text { density }\end{array}$ & T1-hypointense & T2-hypointense \\
\hline $\begin{array}{l}\text { Late subacute } \\
(8 \mathrm{~d}-1 \mathrm{~m})\end{array}$ & $\begin{array}{l}\text { Decreasing } \\
\text { density }\end{array}$ & T1-hyperintense & T1-hyperintense \\
\hline $\begin{array}{l}\text { Chronic } \\
(>1 \mathrm{~m})\end{array}$ & Hypodense & T1-hypointense & $\begin{array}{l}\text { T2-hyperintense } \\
\text { (hypointense rim) }\end{array}$ \\
\hline
\end{tabular}

$h$ hours, $d$ days, $m$ months

\section{Key Point}

- ICH are a frequent cause of severe neurological morbidity and mortality in children. Depending on the etiology and evolutionary phase of the ICH, CT and/ or MRI imaging characteristics change over time.

\subsection{Infections}

Pediatric central nervous system (CNS) infections are high on the list of differential diagnosis when a child present with fever and altered mental status. Neuroimaging is important in the early diagnosis of CNS infections and should be correlated conjunction with laboratory findings. CNS infections may result in significant morbidity and mortality in children $[2,12]$.

Meningitis is the most common form of bacterial infection of the CNS in children. Causative bacteria vary by age and clinical presentation is different for different age groups.
While older children present with fever, headache, photophobia, nausea, vomiting, and meningismus, neonates may present with less obvious vague symptoms. Meningitis is most commonly caused by hematogenous spread from bacterial infections outside of CNS. Less commonly, it develops from an adjacent infective focus, i.e., sinusitis, mastoiditis, or direct invasion after a skull fracture. In general, neuroimaging is not routinely used for the diagnosis of an uncomplicated meningitis, especially if a lumbar puncture already demonstrated an abnormal number of white blood cells in the CSF confirming diagnosis. If there is no complication, neuroimaging may reveal mildly increased meningeal enhancement. Complications of meningitis may however include areas of adjacent vasogenic or cytotoxic edema, focal ischemic injury, CVST, hydrocephalus, and subdural, epidural, or parenchymal abscesses/empyemas. MRI with contrast is the imaging modality of choice to evaluate the complications of bacterial meningitis (Fig. 14.3a-e) [18].

Parenchymal brain infections include cerebritis and cerebral abscess. Symptoms are variable but typically include fever, headache, nausea/vomiting, focal neurological deficits, and seizures. Visual field defects or personality changes can also be seen. If the abscess collection is large, significant mass effect may cause increased intracranial pressure. Neuroimaging is the key to the diagnosis of parenchymal brain infections. MRI with contrast is the most sensitive and specific modality for the radiology diagnosis (Fig. 14.3a-e). Cerebritis may be treated conservatively with intravenous antibiotics, but cerebral abscess requires surgical intervention or drainage [18].

Pyogenic ventriculitis is a life threatening form of CNS infections. The infectious agents are similar to meningitis and abscess. MRI is key to diagnosis; ependymal enhancement after IV contrast injection is usually very prominent. DWI/DTI may show restricted diffusion with 
Fig. 14.2 In this 17-year old boy presenting acutely with ictal headache, vomiting, and bradycardia, axial CT (a) shows fresh hematoma involving the left parietal lobe with contralateral midline shift and intraventricular blood at the foramen of Monro. CT angiography (b) did not reveal abnormal vessels. MRI performed after 12 hours confirms a large intraaxial bleed which is grossly isointense with gray matter on T1-weighted MRI (c) and hyperintense on T2-weighted MRI (d), consistent with a hyperacute hematoma (prevailing oxyhemoglobin)
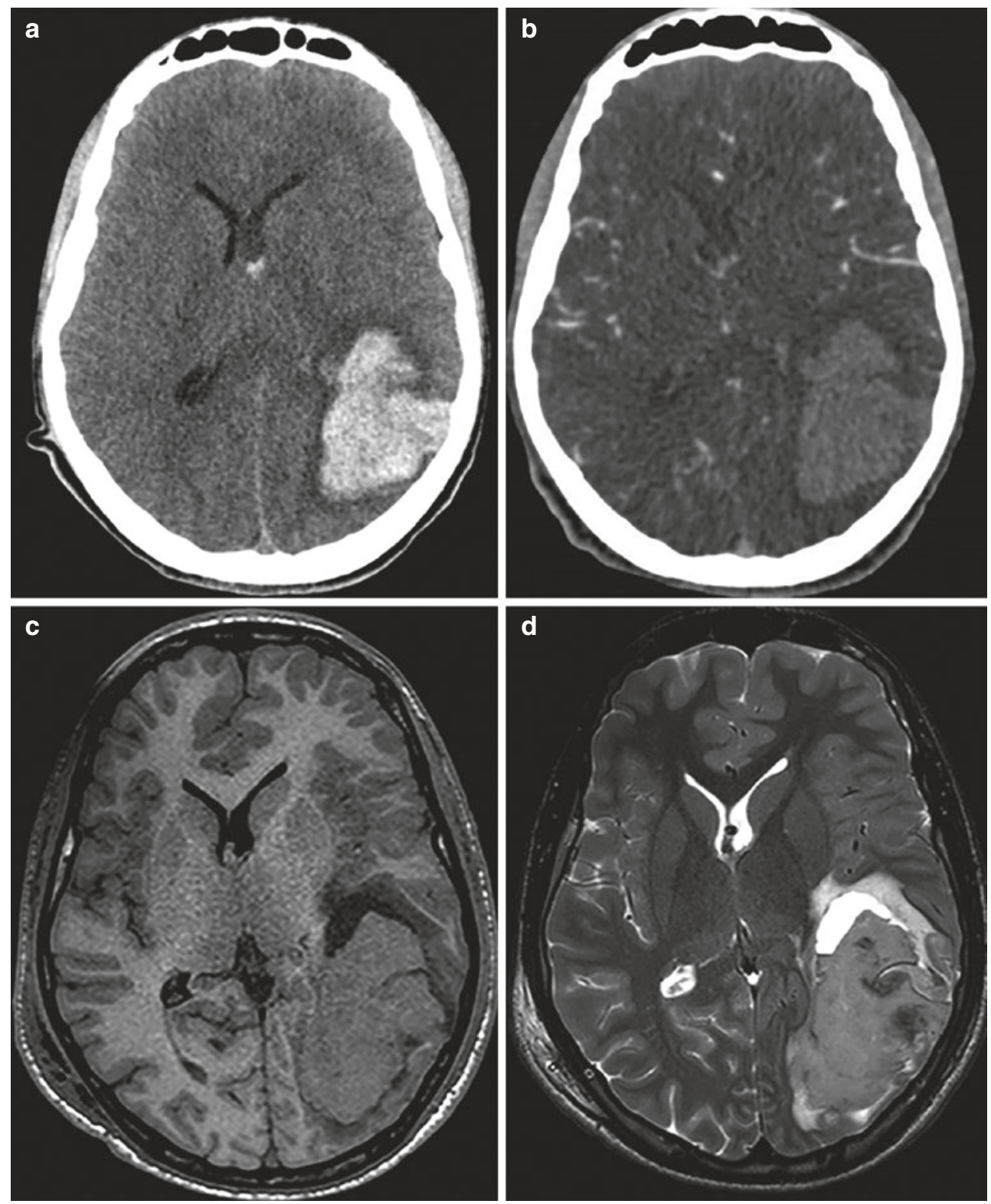

reduced ADC values, especially if pus is collecting within the ventricles [18].

Subdural empyema is an infectious fluid collection between dura and arachnoidea (Fig. 14.3a-e). The acute form develops as a result of spread from, e.g., a paranasal sinus infection and is characterized by high fever, headache, meningismus or focal neurological deficits, more rarely seizures. The complications include CVST, cerebral infarction, cerebral edema, or cerebritis. MRI with contrast is the diagnostic modality of choice [18].

Other acute pediatric CNS infections include: (1) viral meningitis and its complications which include ADEM, transverse myelitis, Guillain-Barré syndrome, and cranial neuritis; (2) fungal CNS infections which are most commonly seen in premature or immunocompromised children; and (3) parasitic CNS infections. Early and correct recognition of the neuroimaging findings of these diseases is critical to guide treatment [19].

\section{Key Point}

- The diagnosis of uncomplicated meningitis is usually confirmed with lumbar puncture by demonstrating an increased number of white blood cells in the CSF. Neuroimaging should be saved to evaluate for life threatening complications of meningitis, including empyema or parenchymal infections. MRI with contrast is the diagnostic modality of choice. 

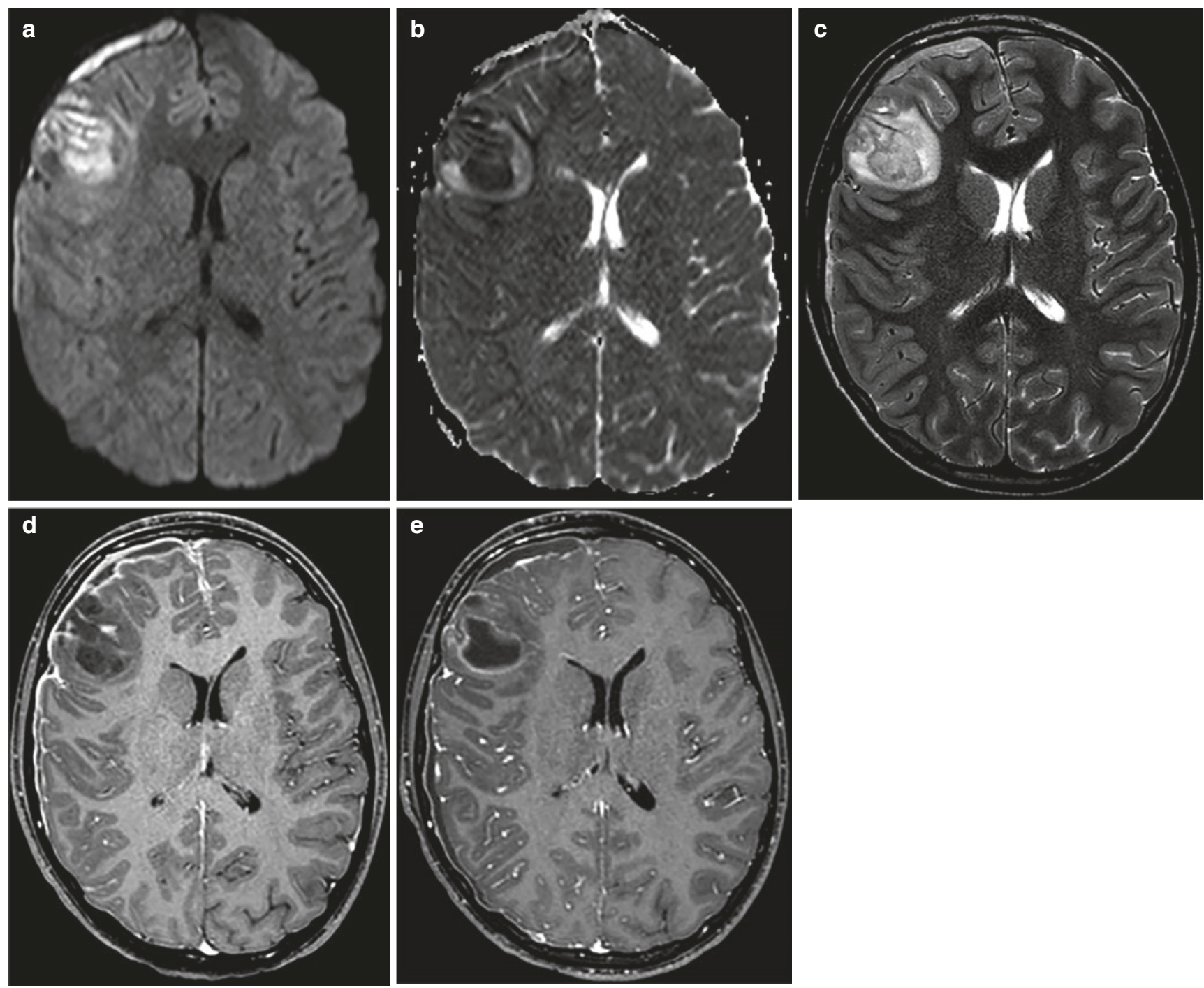

Fig. 14.3 In this 12-year-old boy with suppurative sinusitis (not shown), trace DWI (a) and corresponding ADC map (b) show right frontal subdural empyema and a focal area of restricted diffusion involving the right frontal lobe, corresponding to an ill-defined hyperintense lesion with perifocal edema and mass effect on T2-W MRI (c).
Contrast-enhanced axial T1-weighted MRI (d) shows mild, irregular peripheral enhancement consistent with cerebritis, and also reveals ipsilateral arachnoiditis. Follow-up MRI after 4 days (e) shows development of a well-defined enhancing ring corresponding to evolution towards a frank abscess

\subsection{Trauma}

Traumatic brain injury (TBI) is a significant cause of lifelong disability and mortality in children. The diagnosis of pediatric head trauma requires a multidisciplinary approach. Trauma mechanisms differ from adults and their impact on the developing brain results in unique primary and secondary brain lesions. Birth trauma is responsible for almost all cases of neonatal trauma (parturitional injury); child abuse is a frequent cause of trauma in the first 2 years, whereas falls and motor-vehicle accidents are responsible for the majority of TBI in toddlers and adolescents [20].

Primary TBI lesions include (1) calvarial and maxillofacial fractures; (2) extra-axial: epidural hematoma, subdural hematoma, subarachnoid hemorrhage, and intraventricular hemorrhage; (3) intraaxial: diffuse axonal injury, cortical contusion, and intracerebral hematoma; and (4) vascular: dissection, carotid cavernous fistula, arteriovenous dural fistula, and pseudoaneurysm. Secondary TBI includes both (1) acute: diffuse cerebral edema, brain herniation (subfalcine, uncal, transtentorial), infarction, or infection and (2) chronic injuries: hydrocephalus, encephalomalacia, CSF leak, and leptomeningeal cyst [21]. Different imaging modalities may identify treatable primary TBI and lead to prevention or limitation of secondary TBI. A recommended minimal standard MRI protocol includes (1) a 3D T1-WI, preferably in the sagittal plane, which allows multiplanar reconstruction, (2) an axial T2-WI, (3) an axial DWI/DTI with ADC mapping, 

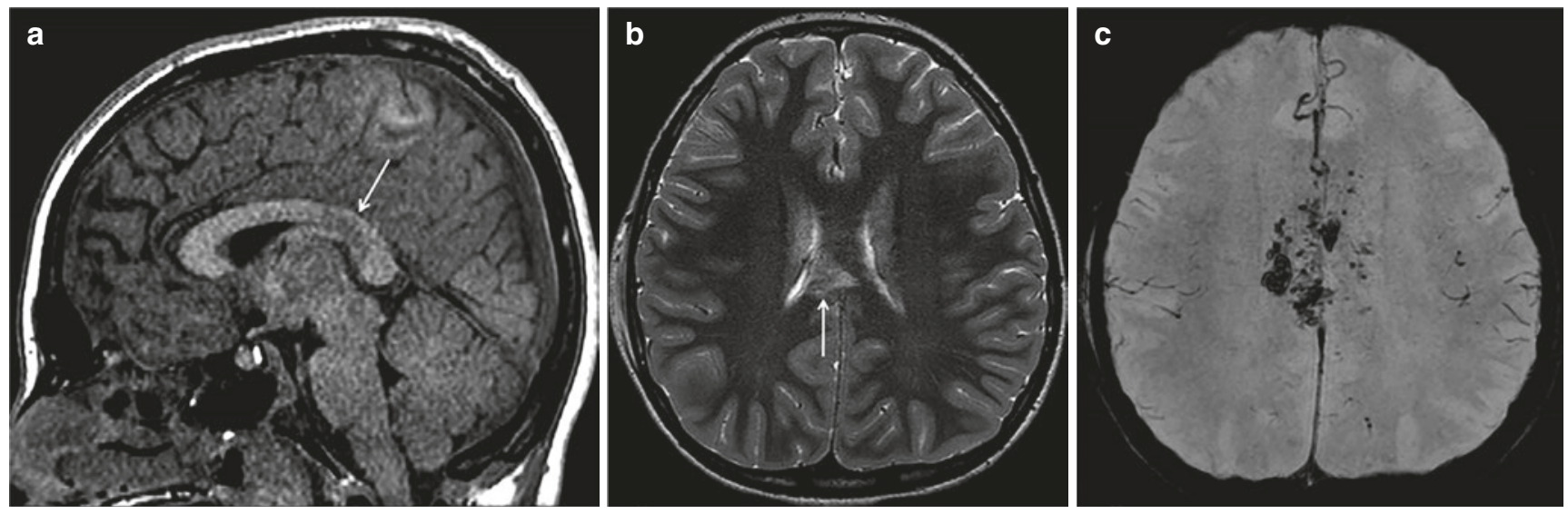

Fig. 14.4 In this 12-year-old boy with a severe blunt head trauma, sagittal T1-weighted MRI (a) and axial T2-W MRI (b) show ill-defined signal abnormality involving the corpus callosum (arrows). SWI (c) reveals more extensive hypointensities consistent with hemorrhagic traumatic axonal injury and (4) an axial SWI from the skull base to the vertex (Fig. 14.4) [20].

It is important to keep child abuse in mind when dealing with trauma in children in the emergency setting. Abusive head trauma diagnosis is challenging both clinically and radiologically; the provided trauma history might not always reflect the truth and no single imaging finding is specific or diagnostic for abusive head trauma by itself. A multiplicity of trauma related findings and severity of findings inconsistent with the provided mechanism of trauma provide clues for the diagnosis. The radiological findings may vary for different types of injury. The common injury mechanisms related to abusive head trauma can be summarized as non-impact shaking injury, direct impact blunt trauma, whiplash-shaking injury of the craniocervical junction, strangulation injury, and stabbing injury. Further diagnostic workup should include a skeletal survey and possible additional cross-sectional studies to exclude other non-CNS solid organ injury $[1,21]$.

Spinal cord injury mechanisms in children differ from adults. Vertebral column fractures and spinal cord injuries are less common in children than adults. Pediatric spinal injuries primarily involve the cervical spine mostly in the proximal part including the craniocervical junction [22]. Thoracolumbar fractures often occur at T11 to L2 levels but may also involve other levels. However, if the injury mechanism is of the seat-belt flexion-distraction type, it typically occurs at L2-L4 levels with high association of accompanying visceral injury. In children of all ages, spinal MRI evaluation is important in the setting of unexplained instability, persistent or delayed symptoms, decreased consciousness, or abnormal X-ray or CT findings. MRI findings including spinal cord transection, intramedullary hemorrhage, edema extending over more than one spinal segment, and persistent compression of the spinal cord are predictive of poor neurological outcome [12].

\section{Key Point}

- TBI is a leading cause of lifelong disability and mortality in children. Birth trauma is responsible for many cases of neonatal TBI; child abuse is a frequent cause of TBI in the first 2 years, falls and motor-vehicle accidents are responsible for the majority of TBI in toddlers and adolescents. CT or MRI are the primary diagnostic imaging modalities of choice depending on the etiology of TBI and the age of the child.

\subsection{Drowning/Near Drowning}

Drowning is a leading cause of accidental death and severe neurologic morbidity. Anoxic brain injury is a common consequence of non-fatal drowning and may cause severe lifelong disability. Irreversible injury develops in the hippocampi, basal ganglia, and cerebral cortex within 4-10 $\mathrm{min}$ following the hypoxic incident. Therefore, early neuroimaging after drowning allows detection of the injury that requires intervention and may also be helpful for the prediction of brain injury severity [23]. CT is usually limited to detect head and neck trauma, but it may detect the loss of gray white matter differentiation and cerebral edema. However a normal looking brain parenchyma on initial/acute CT does not rule out brain injury. MRI especially DWI/DTI with ADC mapping can detect brain injury more reliably, even within minutes after the incident and improves the ability to predict the outcome (Fig. 14.5). Cortical and deep gray matter DWI/ DTI abnormalities and lower ADC values are associated with poor outcomes. 

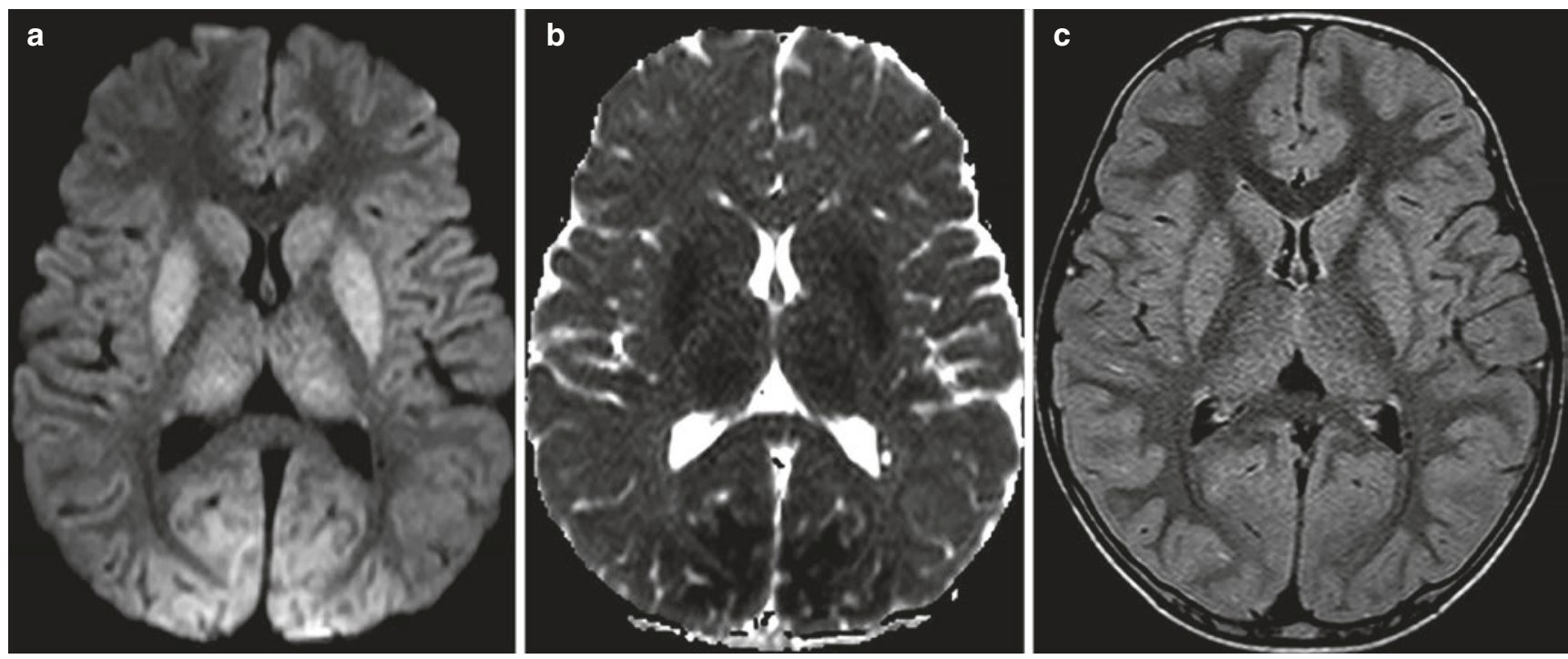

Fig. 14.5 In this 8-year-old boy with severe anoxia due to neardrowning, trace DWI (a) and corresponding ADC map (b) show restricted diffusion involving the basal ganglia, thalami, and parieto-

\section{Key Point}

- The pediatric brain is extremely sensitive to hypoxia and irreversible brain damage develops within 4-10 min after drowning. MRI with DWI/DTI and ADC mapping should be acquired and evaluated promptly for the children with a drowning history.

\subsection{Seizures}

New onset seizures are the most common neurologic emergencies of children presenting to the ER [1,2]. Seizures are abnormal movements or behavior resulting from abnormal electrical activity in the brain. Acute symptomatic seizures occur at the time of a systemic insult or following brain insult with incidence reaching 29-39/100000 per year. Traumatic brain injury, cerebrovascular disease, drug withdrawal, infarction, and metabolic insults are the most common causes $[1,2]$. Unprovoked seizures occur in the absence of precipitating factors, and may be single or recurrent (epilepsy).

A detailed neurological examination with general physical examination and electroencephalogram should be performed initially [1]. Neuroimaging may not be necessary for every child with seizure or epilepsy. For example, neuroimaging is not indicated in children with simple febrile seizures but it should be considered in children with long seizures with focal onset and focal neurological deficits, also it should occipital cortex consistent with cytotoxic edema. Axial FLAIR (c) is relatively unrevealing in the acute phase

be strongly considered in neonates with seizures, because an identifiable cause for seizures can be found in up to $90 \%$ of full term newborns $[1,2]$. When neuroimaging is necessary, MRI is the modality of choice in most of the cases. The aim is to investigate acute focal disorders that need immediate intervention including acute hydrocephalus, acute stroke or hemorrhage, hypoxic ischemic injury in newborns, encephalitis, or tumors. Other relevant disorders include leukodystrophies, metabolic disorders, mesial temporal sclerosis, and malformations [2].

\section{Key Point}

- When dealing with children with seizures in the ER, neuroimaging should be saved for cases with history of long time seizures with focal onset and focal neurological deficits, it should also be strongly considered in neonates with seizures.

\subsection{Autoimmune CNS Diseases with Acute Presentation}

Children with autoimmune CNS diseases may also present to the ER with acute signs and symptoms.

ADEM is a monophasic, immune-mediated demyelinating CNS disorder. Patients usually present 1-3 weeks following an upper respiratory infection or vaccination and may present acutely with significant mass effect due to a tumefactive 

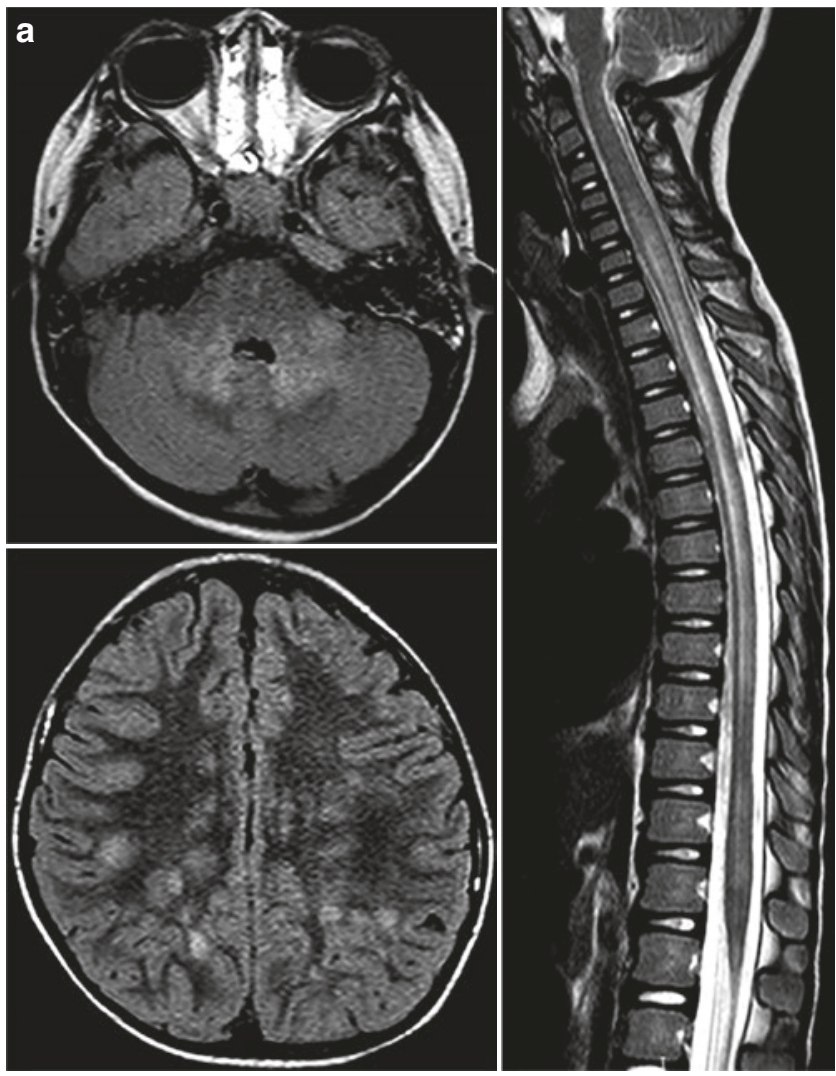

Fig. 14.6 (a) In this 3-year-old girl with a subacute onset of paraparesis and stupor, axial FLAIR shows ill-defined hyperintensities involving the deep cerebellar and supratentorial white matter. Sagittal T2-weighted MRI of the spine shows quasi-holocord swelling and hyperintensity. After treatment with IVIG and steroids, follow-up MRI at 3 months was normalized (not shown), consistent with acute disseminated encephalomyelitis (ADEM). (b) In this 5-year-old girl presenting with headaches and left hemiparesis, trace DWI and corresponding ADC map show a

lesion, with seizures and coma or with behavioral disturbances, headaches, vomiting, fever, or focal neurological signs. Brain MRI typically shows diffuse, poorly demarcated, bilateral, or asymmetrical T2 and FLAIR hyperintense lesions affecting both gray and white matter. The spinal cord may also be involved, showing ill-defined, multiple T2 hyperintense lesions usually involving 2-3 vertebral segments (Fig. 14.6a). ADEM is usually a diagnosis of exclusion.

Multiple sclerosis (MS) is an idiopathic inflammatory disorder characterized by demyelination and degeneration of the CNS, affects most commonly adults, but $3-10 \%$ of patients present their first clinical event during childhood. Pediatric onset MS has higher relapse rates and a less favorable prognosis [24]. Therefore, early diagnosis and prompt treatment of pediatric onset MS is necessary (Fig. 14.6b). Ruling out ADEM and neuromyelitis optica spectrum disorder (NMOSD) is important, because although the clinical presentation of these three disorders
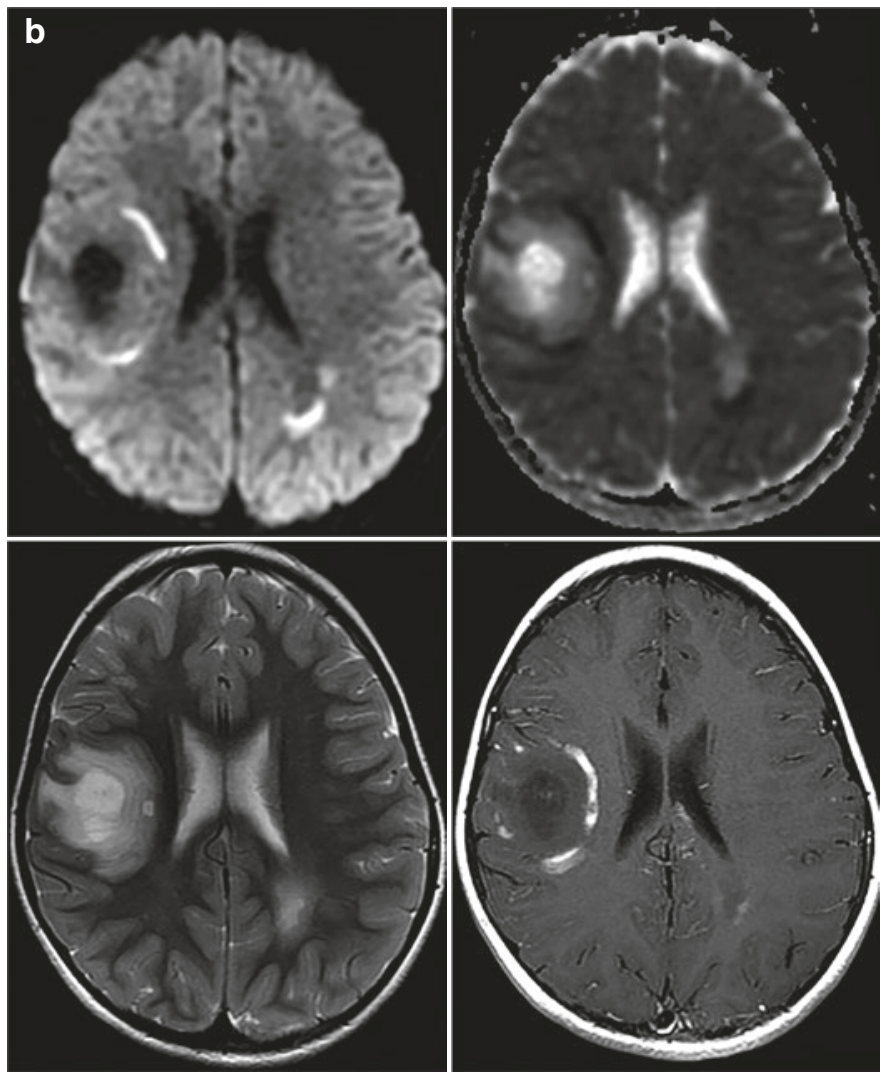

large right inferior frontal lesion with a necrotic center and an incomplete peripheral ring of restricted diffusion. Another small lesion with similar characteristics is seen in the left parietal lobe. On T2-weighted MRI, the larger lesion shows a concentric onion-bulb appearance. Contrast-enhanced axial T1-weighted MRI shows incomplete peripheral ring enhancement corresponding to the areas of restricted diffusion, consistent with tumefactive demyelination

is similar, the treatment is different in each one. Characteristic MRI criteria of MS on brain MRI are helpful to differentiate MS from ADEM and NMOSD but CSF and serum laboratory findings are also required to confirm the diagnosis [25].

NMOSD is an inflammatory demyelinating disease characterized by optic neuritis and acute longitudinally extending myelitis involving at least three vertebral segments. Most clinical, neuroimaging, and laboratory characteristics of pediatric onset NMOSD are similar to those of adult onset disease [26]. Identification of aquaporin-4 immunoglobulin antibodies serves as an important biomarker for the diagnosis. Longitudinally extending myelitis during an acute attack is T2 hyperintense on sagittal spinal cord MRI with central cord predominance and may matching show contrast enhancement on T1-WI. Optic nerve MRI shows unilateral or bilateral T2 hyperintensity or contrast enhancement on T1-WI within the optic 
nerve(s) or optic chiasm. Typical brain lesions are T2 hyperintense involving the dorsal medulla, peri-ependymal surfaces of the fourth ventricle, hypothalamus, thalamus, or peri-ependymal surfaces of third ventricle, as well as large, confluent, unilateral or bilateral subcortical or deep white matter lesions or long, diffuse, heterogeneous corpus callosum lesions or long corticospinal tract lesions [26].

\section{Key Point}

- Children with autoimmune CNS diseases may present to the ER with acute signs and symptoms. In addition to CSF and serum laboratory findings, brain and spinal cord MRI is helpful to differentiate MS from ADEM and NMOSD.

\subsection{Toxic/Metabolic Imbalances}

Diabetic ketoacidosis (DKA) is an acute serious complication in children with diabetes mellitus type 1 . This state may cause significant long-term neurological morbidity and mortality. Brain edema is the most common neuroimaging finding in children with DKA and may cause brain herniation with subsequent cerebral infarctions due to vascular compression. Also, primary ischemic or hemorrhagic stroke during the acute DKA episode may be seen in $10 \%$ of cases [27].

Neonatal hypoglycemia is an important issue in term neonates and can cause severe brain injury. Neuroimaging typically shows focal edema or infarcts most commonly in bilateral parieto-occipital lobes, basal ganglia, corpus callosum, and thalami [12]. Because the urine strip test diagnosis of hypoglycemia can be made simply, neuroimaging is usually used to evaluate the degree of brain injury and estimation of prognosis rather than to confirm the diagnosis.

Osmotic demyelination syndrome is an acute demyelination process that usually occurs several days after a rapid change in serum osmolality and is uncommon in children. It can present with central pontine myelinolysis or extrapontine myelinolysis. MRI is the diagnostic modality of choice. Central pontine myelinolysis is seen with characteristic trident-shaped, bat-winged, or piglet hyperintensity on T2-WI, hypointensity on T1-WI with no mass effect in the central pons, with sparing of the ventrolateral pons, tegmentum, and corticospinal tracts. Extrapontine lesions are usually bilateral and most commonly seen in the cerebellar peduncles, globus pallidus, thalamus, lateral geniculate body, putamen, external and extreme capsule, splenium of corpus callosum, and supratentorial white matter [28].

\section{Key Point}

- Neuroimaging with MRI may be necessary when dealing with children with metabolic imbalances in the ER when brain edema is suspected. In addition, MRI is the diagnostic modality of the choice to evaluate the osmotic demyelination syndrome.

\subsection{Hydrocephalus}

Hydrocephalus in children could be either due to overproduction of CSF by a tumor, ventricular obstruction, or dysfunctional Pachionic granulations along the subarachnoid space. Children can present to the ER with (1) acute findings of hydrocephalus or (2) complications of shunted hydrocephalus including malfunction and infection.

Clinical presentation of hydrocephalus varies with age; in infants abnormally rapidly increasing head circumference, irritability, vomiting, bulging of the fontanelle; in older children headache, vomiting, diplopia, or papilledema can be seen [29]. Neuroimaging should be directed to identify the underlying cause. MRI is the imaging modality of choice in suspected hydrocephalus cases. Next to axial T1-WI or T2-WI, a thin section, high resolution, T2-WI in sagittal plane is the most helpful MRI sequence to evaluate these cases $[1,2]$. Contrast administration would be necessary if a tumor is suspected.

Shunt malfunction is the most common complication of ventricular shunts. Children present with headache, nausea, vomiting, and altered mental status. Multiple radiographs of the skull, neck, chest, and abdomen to evaluate the shunt integrity are often the initial imaging in some institutions $[1,2]$. CT is often used as primary cross-sectional imaging because it is readily available, able to detect changes in the ventricular size and configuration, identify intracranial hemorrhages or infarction, shunt catheter discontinuity and catheter migration [2]. Scout views should not be ignored, because occasionally, axial or reconstructed cross-sectional CT images may miss relevant findings. A possible disconnection of a shunt may be within the neck region below or outside of the scanned region; however, the information may be visible on the scout view which is a standard component of each CT study [30]. Children with infection due to a shunted hydrocephalus present with altered mental status, fever, nausea, and vomiting. CSF cultures are usually sufficient for the diagnosis 
in almost $95 \%$ of the cases and neuroimaging has a limited role in the diagnosis [1]. Uncommonly, infection presents with abdominal symptoms from a peritoneal CSF pseudocyst. Abdominal ultrasound or CT is useful to evaluate these cases [2].

\section{Key Point}

- Children could present to the ER with acute findings of hydrocephalus or complications of shunted hydrocephalus including shunt malfunction and infection. MRI or CT should be used to differentiate the etiology.

\subsection{Concluding Remarks}

Children with acute neurological emergency present to the ER with a wide spectrum of symptoms and signs. Neuroimaging is often needed in children with acute neurological emergency due to the difficulties of gathering a good and reliable patient history or limited physical/neurological examination. The radiologist should be able to answer if the child needs neuroimaging emergently, and which imaging modality is appropriate for the child. Different modalities may be chosen based on the institutional availability and clinical status of the patient. CT and MRI are the most frequently used imaging modalities in the emergency setting. Finally, the prompt diagnosis is essential when dealing with children with acute neurological emergency especially in the time sensitive pathologies, i.e., stroke, drowning.

\section{Take Home Messages}

- Radiology is an integral part of the ER.

- Children, especially infants and young children may present to the ER with non-specific symptoms or subtle findings.

- The threshold for neuroimaging should be held low especially for infants and young children.

- Neuroimaging should be promptly acquired and evaluated when dealing with children with acute neurological emergencies in the ER.

\section{References}

1. Saigal G, Ezuddin NS, Vega G. Neurologic emergencies in pediatric patients including accidental and nonaccidental trauma. Neuroimaging Clin N Am. 2018;28(3):453-70. https://doi. org/10.1016/j.nic.2018.03.007.

2. Prabhu SP, Young-Poussaint T. Pediatric central nervous system emergencies. Neuroimaging Clin N Am. 2010;20(4):663-83. https://doi.org/10.1016/j.nic.2010.07.008.

3. Chang PT, Yang E, Swenson DW, Lee EY. Pediatric emergency magnetic resonance imaging: current indications, techniques, and clinical applications. Magn Reson Imaging Clin N Am. 2016;24(2):449-80. https://doi.org/10.1016/j.mric.2015.11.009.

4. Singh RK, Smith JT, Wilkinson ID, Griffiths PD. Ultrafast MR imaging in pediatric neuroradiology. Acta Radiol. 2003;44(5):550 7. https://doi.org/10.1034/j.1600-0455.2003.00118.x.

5. Stacey A, Toolis C, Ganesan V. Rates and risk factors for arterial ischemic stroke recurrence in children. Stroke. 2018;49(4):842-7. https://doi.org/10.1161/STROKEAHA.117.020159.

6. Goeggel Simonetti B, Cavelti A, Arnold M, Bigi S, Regenyi M, Mattle HP, et al. Long-term outcome after arterial ischemic stroke in children and young adults. Neurology. 2015;84(19):1941-7. https://doi.org/10.1212/WNL.0000000000001555.

7. Mackay MT, Wiznitzer M, Benedict SL, Lee KJ, Deveber GA, Ganesan V. Arterial ischemic stroke risk factors: the international pediatric stroke study. Ann Neurol. 2011;69(1):130-40. https://doi. org/10.1002/ana.22224.

8. Lee S, Mirsky DM, Beslow LA, Amlie-Lefond C, Danehy AR, Lehman L, et al. Pathways for neuroimaging of neonatal stroke. Pediatr Neurol. 2017;69:37-48. https://doi.org/10.1016/j. pediatrneurol.2016.12.008.

9. Gadian DG, Calamante F, Kirkham FJ, Bynevelt M, Johnson CL, Porter DA, et al. Diffusion and perfusion magnetic resonance imaging in childhood stroke. J Child Neurol. 2000;15(5):279-83.

10. Meoded A, Poretti A, Benson JE, Tekes A, Huisman TA. Evaluation of the ischemic penumbra focusing on the venous drainage: the role of susceptibility weighted imaging (SWI) in pediatric ischemic cerebral stroke. J Neuroradiol. 2014;41(2):108-16. https://doi. org/10.1016/j.neurad.2013.04.002.

11. Shack M, Andrade A, Shah-Basak PP, Shroff M, Moharir M, Yau I, et al. A pediatric institutional acute stroke protocol improves timely access to stroke treatment. Dev Med Child Neurol. 2017;59(1):317. https://doi.org/10.1111/dmcn.13214.

12. Lall NU, Stence NV, Mirsky DM. Magnetic resonance imaging of pediatric neurologic emergencies. Top Magn Reson Imaging. 2015;24(6):291-307. https://doi.org/10.1097/ RMR.0000000000000068.

13. Jordan LC, Hillis AE. Challenges in the diagnosis and treatment of pediatric stroke. Nat Rev Neurol. 2011;7(4):199-208. https://doi. org/10.1038/nrneurol.2011.23.

14. Shellhaas RA, Smith SE, O'Tool E, Licht DJ, Ichord RN. Mimics of childhood stroke: characteristics of a prospective cohort. Pediatrics. 2006;118(2):704-9. https://doi.org/10.1542/peds.2005-2676.

15. Meyer-Heim AD, Boltshauser E. Spontaneous intracranial haemorrhage in children: aetiology, presentation and outcome. Brain Dev. 2003;25(6):416-21. https://doi.org/10.1016/ S0387-7604(03)00029-9. 
16. Huisman TA, Singhi S, Pinto PS. Non-invasive imaging of intracranial pediatric vascular lesions. Childs Nerv Syst. 2010;26(10):127595. https://doi.org/10.1007/s00381-010-1203-1.

17. Langford S, Panigrahy A, Narayanan S, Hwang M, Fitz C, Flom L, et al. Multiplanar reconstructed CT images increased depiction of intracranial hemorrhages in pediatric head trauma. Neuroradiology. 2015;57(12):1263-8. https://doi.org/10.1007/s00234-015-1584-7.

18. Nickerson JP, Richner B, Santy K, Lequin MH, Poretti A, Filippi CG, et al. Neuroimaging of pediatric intracranial infection--part 1: techniques and bacterial infections. J Neuroimaging. 2012;22(2):e4251. https://doi.org/10.1111/j.1552-6569.2011.00700.x.

19. Nickerson JP, Richner B, Santy K, Lequin MH, Poretti A, Filippi $\mathrm{CG}$, et al. Neuroimaging of pediatric intracranial infection--part 2: TORCH, viral, fungal, and parasitic infections. J Neuroimaging. 2012;22(2):e52-63. https://doi. org/10.1111/j.1552-6569.2011.00699.x.

20. Pinto PS, Poretti A, Meoded A, Tekes A, Huisman TA. The unique features of traumatic brain injury in children. Review of the characteristics of the pediatric skull and brain, mechanisms of trauma, patterns of injury, complications and their imaging findings--part 1. J Neuroimaging. 2012;22(2):e1-e17. https://doi. org/10.1111/j.1552-6569.2011.00688.x.

21. Pinto PS, Meoded A, Poretti A, Tekes A, Huisman TA. The unique features of traumatic brain injury in children. Review of the characteristics of the pediatric skull and brain, mechanisms of trauma, patterns of injury, complications, and their imaging findings--part 2. J Neuroimaging. 2012;22(2):e18-41. https://doi. org/10.1111/j.1552-6569.2011.00690.x.

22. Meoded A, Singhi S, Poretti A, Eran A, Tekes A, Huisman TA. Tectorial membrane injury: frequently overlooked in pediatric traumatic head injury. AJNR Am J Neuroradiol. 2011;32(10):180611. https://doi.org/10.3174/ajnr.A2606.

23. Topjian AA, Berg RA, Bierens JJ, Branche CM, Clark RS, Friberg $\mathrm{H}$, et al. Brain resuscitation in the drowning victim. Neurocrit Care. 2012;17(3):441-67. https://doi.org/10.1007/s12028-012-9747-4.

24. Tenembaum SN. Pediatric multiple sclerosis: distinguishing clinical and MR imaging features. Neuroimaging Clin N Am. 2017;27(2):229-50. https://doi.org/10.1016/j.nic.2016.12.007.

25. Filippi M, Rocca MA, Ciccarelli O, De Stefano N, Evangelou N, Kappos L, et al. MRI criteria for the diagnosis of multiple sclerosis: MAGNIMS consensus guidelines. Lancet Neurol. 2016;15(3):292303. https://doi.org/10.1016/S1474-4422(15)00393-2.

26. Wingerchuk DM, Banwell B, Bennett JL, Cabre P, Carroll W, Chitnis T, et al. International consensus diagnostic criteria for neuromyelitis optica spectrum disorders. Neurology. 2015;85(2):17789. https://doi.org/10.1212/WNL.0000000000001729.

27. Barrot A, Huisman TA, Poretti A. Neuroimaging findings in acute pediatric diabetic ketoacidosis. Neuroradiol J. 2016;29(5):317-22.

28. Bansal LR, Zinkus T. Osmotic demyelination syndrome in children. Pediatr Neurol. 2019;97:12-7. https://doi.org/10.1016/j. pediatrneurol.2019.03.018.

29. Kahle KT, Kulkarni AV, Limbrick DD Jr, Warf BC. Hydrocephalus in children. Lancet. 2016;387(10020):788-99. https://doi. org/10.1016/S0140-6736(15)60694-8.

30. Orman G, Bosemani T, Tekes A, Poretti A, Huisman TA. Scout view in pediatric $\mathrm{CT}$ neuroradiological evaluation: do not underestimate! Childs Nerv Syst. 2014;30(2):307-11. https://doi.org/10.1007/ s00381-013-2288-0.

Open Access This chapter is licensed under the terms of the Creative Commons Attribution 4.0 International License (http://creativecommons. org/licenses/by/4.0/), which permits use, sharing, adaptation, distribution and reproduction in any medium or format, as long as you give appropriate credit to the original author(s) and the source, provide a link to the Creative Commons license and indicate if changes were made.

The images or other third party material in this chapter are included in the chapter's Creative Commons license, unless indicated otherwise in a credit line to the material. If material is not included in the chapter's Creative Commons license and your intended use is not permitted by statutory regulation or exceeds the permitted use, you will need to obtain permission directly from the copyright holder. 